\title{
Search for charged lepton flavour violation with the MEG and MEG II experiments
}

\author{
Marco Venturini ${ }^{1,2, a}$, on behalf of the MEG collaboration \\ ${ }^{1}$ Scuola Normale Superiore - Piazza dei Cavalieri 7, 56126 Pisa, Italy \\ 2 INFN, Sezione di Pisa - Largo B. Pontecorvo 3, 56127 Pisa, Italy
}

\begin{abstract}
.
In the next decade Charged Lepton Flavour Violation (CLFV) experiments will play a key role in the search for new physics. Given that flavour oscillations occur among neutrinos, unmeasurably small amplitudes are expected for CLFV processes, while in many new physics scenarios such processes are strongly enhanced. A new generation of experiments looking for CLFV muon decays will be performed at the Paul Scherrer Institut (MEG II, Mu3e), at Fermilab (Mu2e) and at J-Parc (DeeMee, COMET), with sensitivities improved by one or two order of magnitudes with respect to current limits. The MEG experiment has performed the most recent improvement in the search for the CLFV decay $\mu^{+} \rightarrow e^{+} \gamma$. With the analysis of half of the collected statistics, a new upper limit on the branching ratio has been set $\mathcal{B R}(\mu \rightarrow$ e $\gamma)<5.7 \times 10^{-13}$ at $90 \% \mathrm{CL}$, and the final result is soon to be released. A substantial improvement of MEG results requires an improvement of detector performances, in order to reject the background contributions which limit the signal sensitivity. This will be carried out by a short-term upgrade of the apparatus, MEG II. The major modifications of the experimental apparatus consist in the replacement of the current positron tracking system with a new high transparency and high granularity spectrometer and the substitution and rearrangement of some of the photosensors in the liquid-xenon photon detector. MEG II will start taking data in 2016, with a sensitivity on the $\mu^{+} \rightarrow e^{+} \gamma$ decay of about $5 \times 10^{-14}$. Therefore as MEG performed the last improvement in the search for CLFV, MEG II will start a new era probing unexplored regions of new physics scenarios.
\end{abstract}

\section{Introduction}

The large number of experimental evidences for neutrino oscillations supports that lepton flavour is violated in the neutral sector and thus nonvanishing amplitudes are expected at loop level for flavour changing processes among charged leptons. However charged lepton flavour violation (CLFV) is very small in the Standard Model (SM): for instance the $\mu \rightarrow e \gamma$ decay is strongly suppressed by the fourth power of the the ratio between the neutrino and the $\mathrm{W}$ boson masses [1]. With an expected branching ratio of about $10^{-54}$, this makes Standard-Model CLFV non detectable. The $\mu \rightarrow e \gamma$ process is strictly related to two other CLFV processes: $\mu \rightarrow$ eee and coherent muon-to-electron conversion in muonic atoms. For both processes the SM prediction [2] is below $10^{-50}$. Nevertheless in many new physics

\footnotetext{
a e-mail: marco.venturini@pi.infn.it
} 

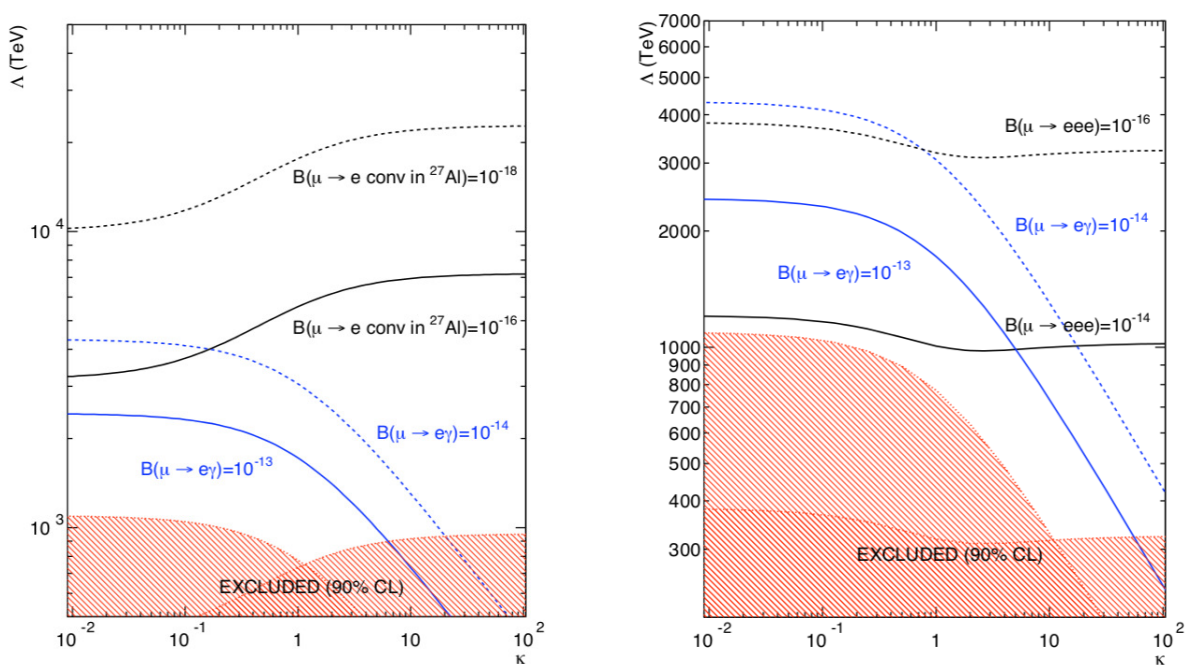

Figure 1. Sensitivities in $\mu \rightarrow e \gamma, \mu \rightarrow e e e$ and $\mu-e$ conversion plotted in the $(\Lambda, \kappa)$ plane [3].

scenarios much larger amplitudes are foreseen. For instance in supersymmetric models, the large mixings occurring among superparticles enhance the $\mathcal{B}(\mu \rightarrow$ eq $)$ up to $10^{-14} \div 10^{-12}$. To evaluate the deep interconnection among muon CLFV channels we can use the following effective Lagrangian [3]

$$
\mathcal{L}=\frac{m_{\mu}}{(\kappa+1) \Lambda^{2}} \bar{\mu}_{R} \sigma_{\alpha \beta} e_{L} F^{\alpha \beta}+\frac{\kappa}{(\kappa+1) \Lambda^{2}} \bar{\mu}_{R} \gamma_{\alpha} e_{L} \bar{f} \gamma^{\alpha} f+\text { h.c. }
$$

where $\kappa$ is an adimensional parameter and $\Lambda$ represents the energy scale of CLFV physics; $m_{\mu}$ is the muon mass, $F^{\alpha \beta}$ is the photon filed strength and $f$ represents a general fermion that is an electron in $\mu \rightarrow e e e$ or a quark in $\mu-e$ conversion. We recognise a dipole term, which powers $\mu \rightarrow e \gamma$ directly and $\mu \rightarrow e e e$ and $\mu-e$ conversion at order $\alpha$, and a four-fermion contact term which mediates only the non-radiative processes. Muon $g-2$ anomaly can be taken into account by adding an additional term, analogous to the first in equation (1) with $\left(\bar{\mu}_{R}, e_{L}\right)$ replaced with $\left(\bar{\mu}_{R}, \mu_{L}\right)$. Figure 1 shows the parameters space that can be explored for a given sensitivity in the three CLFV channels. Note that SM extensions do not populate the plane evenly, so different models enhance the processes in different ways.

The current upper limits on such processes are [4-6]

$$
\begin{aligned}
\mathcal{B}(\mu \rightarrow e \gamma)<5.7 \times 10^{-13}(90 \% \text { C.L. }) & \text { from MEG } \\
\mathcal{B}(\mu \rightarrow e e e)<1.0 \times 10^{-12}(90 \% \text { C.L. }) & \text { from SINDRUM } \\
C \mathcal{R}(\mu \mathrm{Au} \rightarrow e \mathrm{Au})<7 \times 10^{-13}(90 \% \text { C.L. }) & \text { from SINDRUM II }
\end{aligned}
$$

where for muon conversion the measured quantity is the ratio to nuclear capture of muons $C R$. It is clear that the search of all three CLFV processes is important in order to understand the mechanisms beyond lepton flavour violation. The MEG experiment searches for $\mu \rightarrow e \gamma$ at the Paul Scherrer Institut (PSI) in Switzerland, while other experiments in the USA, Europe and Japan are planned to search for $\mu \rightarrow e e e$ (Mu3e at PSI) and $\mu-e$ conversion (Mu2e at Fermilab, Comet at J-Park). 


\section{The MEG experiment}

\subsection{The $\mu \rightarrow$ ey decay}

The $\mu^{+} \rightarrow e^{+} \gamma$ process is a two-body decay: in the reference frame where the $\mu^{+}$is at rest the positron and the photon are emitted back-to-back, with the same energy $\left(\sim m_{\mu} / 2\right)$ and simultaneously. Two major backgrounds can mimic the signal signature: radiative muon decays (RMD) in which neutrinos carry little energy (physics background) and random coincidences of uncorrelated positrons and photons (accidental background). In RMDs, positron and photon observables have continuous distributions whose limits are the values expected for the signal. The number of observed events is given by the integral of the RMD differential branching ratios around the signal region of the phase space for a width given by detector resolutions. However in MEG (and in general in the most recent experiments) the RMD accounts only for the $10 \%$ of the background events. The major contribution comes from accidental detection of a positron and a photon originated from uncorrelated processes. Positrons are mostly produced via Michel decay, while photons with an energy of about $\sim m_{\mu} / 2$ can come from RMDs, positron annihilation-in-flight and bremsstrahlung.

\subsection{The MEG detector}

The MEG apparatus, sketched in Figure 2, is tailored to take advantage of the well-defined kinematics of the $\mu \rightarrow$ er process [7]. The $\pi \mathrm{E} 5$ muon beam line delivers in MEG $3 \times 10^{7} \mu^{+} / \mathrm{s}$ with an average momentum of $28 \mathrm{MeV} / c$. The beam is stopped in a $205 \mu$ m polyethylene target: the emerging positrons are tracked by a magnetic spectrometer composed of a set of drift chamber modules and timing counters, immersed in a non-uniform magnetic field provided by the COBRA superconducting magnet. Photons energy, time and conversion point are measured by a liquid xenon detector.

The non-uniformity of the COBRA magnetic field reduces the dependence of the positron bending radius on the emission angle (COnstant Bending RAdius) and strongly reduces the occupancy of the drift chambers by sweeping away particles with low longitudinal momentum. The drift chamber system is composed of 16 trapezoidal modules, placed in a half circle with radial orientation. Each module contains two independent detector planes, made of an array of alternating field and sense
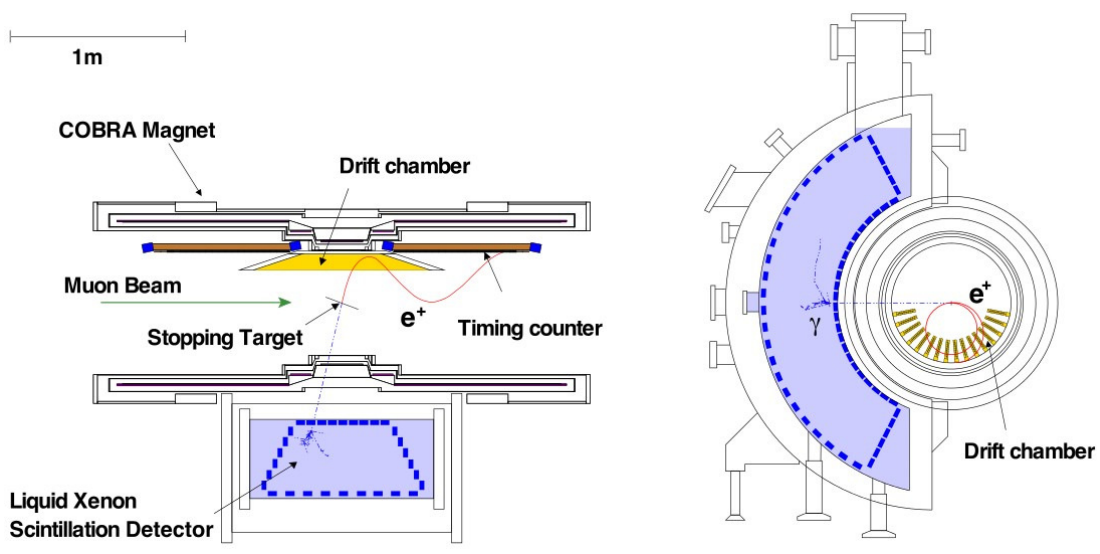

Figure 2. Schematic view of the MEG experiment with a signal event displayed. 
Table 1. Measured resolutions for MEG and expected for MEG II. $(\hat{u}, \hat{v}, \hat{w})$ is the reference system in which the calorimeter is a parallelepiped with $\hat{u}\|\hat{z}, \hat{v}\| \hat{\varphi}$ and $\hat{w} \|-\hat{r}$.

\begin{tabular}{lcc}
\hline Variable & MEG & MEG II \\
\hline$\sigma E_{\gamma}(\%)$ & 2 & 1.0 \\
$\gamma$ position $(\mathrm{mm})$ & $5(u, v), 6(w)$ & $2.4(u), 2.2(v), 3.1(w)$ \\
$\sigma P_{e}(\mathrm{keV})$ & 306 & 130 \\
$e^{+}$angle $(\mathrm{mrad})$ & $8.4\left(\varphi_{e}\right), 9.4\left(\vartheta_{e}\right)$ & $5.3\left(\varphi_{e}\right), 4.8\left(\vartheta_{e}\right)$ \\
$\sigma t_{e \gamma}(\mathrm{ps})$ & 122 & 84 \\
\hline$e^{+}$efficiency $(\%)$ & 40 & 88 \\
$\gamma$ efficiency $(\%)$ & 63 & 69 \\
trigger efficiency $(\%)$ & 99 & 99 \\
\hline
\end{tabular}

wires enclosed by two cathode foils each, equipped with Vernier pads ${ }^{1}$ for the determination of the position of the hit along the wire. The modules are filled with a 50:50 helium-ethane gas mixture, while the remaining volume inside COBRA is filled with pure helium. The single-hit resolution, i.e. the accuracy in the determination of the impact parameter on a wire, is about $200 \mu \mathrm{m}$. The resolution on the positron angle is determined from data of events where the positron makes two turns in the drift chamber system. The obtained values are $\sigma_{\vartheta_{e}} \sim 9.4 \mathrm{mrad}$ and $\sigma_{\varphi_{e}} \sim 8.4 \mathrm{mrad}$. The energy resolution of the drift chamber is $330 \mathrm{keV}$, measured by fitting the measured Michel spectrum.

Two identical hodoscopes (timing counters) are placed at the two sides of the drift chambers along the beam axis for the determination of positron time. Each hodoscope consists of a layer of scintillating fibres, read out by avalanche photodiodes, placed on the top of an array of 15 scintillating bars, which are coupled to fine-mesh fast photomultipliers. Bars and fibres are oriented perpendicularly to each other, with the bars being parallel to the beam axis. The timing resolution of the detector is about $70 \mathrm{ps}$, determined through tracks hitting on multiple bars.

The photon detector is a homogeneous calorimeter with 900 litres of liquid xenon viewed by 846 vacuum-ultra-violet sensitive photomultipliers, and placed in a cryostat that keeps xenon temperature stable at about $165 \mathrm{~K}$. The small radiation length $\left(X_{0}=2.7 \mathrm{~cm}\right)$ and the high light-yield (about 40 scintillation photons per $\mathrm{keV}$ of deposited energy) confer to liquid xenon high detection efficiency, small leakage for electromagnetic showers and good energy resolution. In addition, all the three main mechanisms involved in scintillation have time constants below $50 \mathrm{~ns}$. Such a fast response results in reduced pile-up probability and in a superior timing resolution (better than $100 \mathrm{ps}$ ). Energy and timing resolution of the calorimeter are extracted from the calibrations. Energy resolution depends on the position of $\gamma$-ray conversion: it is about $2 \%$ for conversions within $3 \mathrm{~cm}$ from the inner face and $1.6 \%$ for deeper conversions. The acceptance for photons emerging from the target is about $11 \%$, and this sets the acceptance of the apparatus.

For the measurement and the monitor of the apparatus performance a rich set of calibrations has been developed. They involve both single detectors (energy scale, resolution variations...) and their intercalibration. They are performed with not only the muon beam but also with a positron beam, cosmic rays and gamma lines resulting from nuclear interactions induced by protons from a CockcroftWalton accelerator. A special calibration is performed with charge exchange reactions $\pi^{-} p \rightarrow \pi^{0}(\gamma \gamma) n$ from a $\pi^{-}$beam (obtained changing the settings of the $\pi E 5$ beam line) stopped in a liquid hydrogen target which temporarily replaces the polyethylene one. Photons from $\pi^{0}$ decays are used to calibrate

\footnotetext{
${ }^{1}$ each cathode foil has a periodic double-wedge structure with the two half-foils (Vernier pads) having independent readout, so that the measurement of the asymmetry in charge collection on the two pads conveys the information on the $z$-coordinate. In the two cathode foils enclosing a plane of the detector, the patterns are displaced by a quarter-period.
} 
the response of the xenon detector at an energy $(55 \mathrm{MeV})$ close to that of signal photons. The overall resolutions of the MEG detector on the kinematic variables are reported in Table 1.

In MEG the trigger is implemented in a cascaded custom made VME boards, performing a basic event reconstruction with signals from xenon detector and timing counters, digitized by a $100-\mathrm{MHz}$ ADC. Signals from the detectors are digitized by a waveform digitizer chip, the Domino Ring Sampler 4 (DRS4) [8]. This is necessary to exploit the detectors timing resolutions and to reduce the pile up of events.

\subsection{The analysis}

For the measurement of the branching ratio of the process a likelihood analysis is performed [4]. The distribution of events is studied in terms of 5-dimensional vectors $\vec{x}=\left(E_{\gamma}, E_{e}, t_{e \gamma}, \vartheta_{e \gamma}, \varphi_{e \gamma}\right)$. The analysis follows a blinding procedure: events falling in a blinding window containing the signal region (48 MeV $<E_{\gamma}<58 \mathrm{MeV}$ and $\left|t_{e \gamma}\right|<1 \mathrm{~ns}$ ) are written in separate files which are not opened until the analysis procedure is completely defined.

The first step of the analysis is the determination of the Probability Density Functions (PDFs) for signal $S(\vec{x})$, radiative backgrounds $R(\vec{x})$ and accidental background $B(\vec{x})$, using both calibration data and distributions in the sidebands, which are the regions of the $\left(t_{e \gamma}, E_{\gamma}\right)$ plane next to the blinding box. Once the PDFs are determined, the number of signal, RMD and accidental events $\left(N_{\mathrm{sig}}, N_{\mathrm{RMD}}, N_{\mathrm{ACC}}\right)$ is extracted by maximizing the following likelihood function:

$$
\begin{aligned}
\mathcal{L}\left(N_{\text {sig }}, N_{\mathrm{RMD}}, N_{\mathrm{ACC}}\right) & =\frac{e^{-N}}{N_{\mathrm{obs}} !} \prod_{i=1}^{N_{\mathrm{obs}}}\left[N_{\text {sig }} S\left(\vec{x}_{i}\right)+N_{\mathrm{RMD}} R\left(\vec{x}_{i}\right)+\operatorname{NathrmACCB}\left(\vec{x}_{i}\right)\right] \times \\
& \times \exp \left[-\frac{\left(N_{\mathrm{RMD}}-\left\langle N_{\mathrm{RMD}}\right\rangle\right)^{2}}{2 \sigma_{\mathrm{RMD}}^{2}}\right] \exp \left[-\frac{\left(N_{\mathrm{ACC}}-\left\langle N_{\mathrm{ACC}}\right\rangle\right)^{2}}{2 \sigma_{\mathrm{ACC}}^{2}}\right]
\end{aligned}
$$

where $N_{\text {obs }}$ is the number of events detected in the signal window and $N=N_{\text {sig }}+N_{\text {RMD }}+N_{\mathrm{ACC}}$. Estimates of the number of background events in the signal region $\left\langle N_{\mathrm{RMD}, \mathrm{ACC}}\right\rangle$ with corresponding variances $\sigma_{\mathrm{RMD}, \mathrm{ACC}}^{2}$ are obtained from the sidebands. This additional measurement is included in the analysis by means of the two Gaussian terms in the likelihood function. Figure 3 shows the likelihood (blue solid line) fit projected on the five kinematic variables. Individual best fits for signal (green), RMD (red) and accidental background (magenta) are reported.

For the determination of the branching ratio, the number of measured events is normalized to the numbers of measured Michel positrons and RMDs events. The combination of these two independent methods leads to a $4 \%$ uncertainty in the branching ratio estimate. The computation of the confidence interval is based on frequentist method with a profile likelihood-ratio ordering, where the number of RMD events and the number of accidental events are treated as nuisance parameters.

With the analysis of data collected in the period 2009-2011, an upper limit on the branching ratio of $5.7 \times 10^{-13}$ was obtained at $90 \%$ of confidence level (CL). The analysis of the full set of data collected in the period 2009-2013, with a double statistics, is close to completion, with a sensitivity of $4 \times 10^{-13}$ on the branching ratio at $90 \% \mathrm{CL}$. However a substantial improvement of MEG results requires an improvement of detector performances, in order to reject the background contributions which limit the signal sensitivity. This will be carried out by a short-term upgrade of the apparatus, MEG II. 

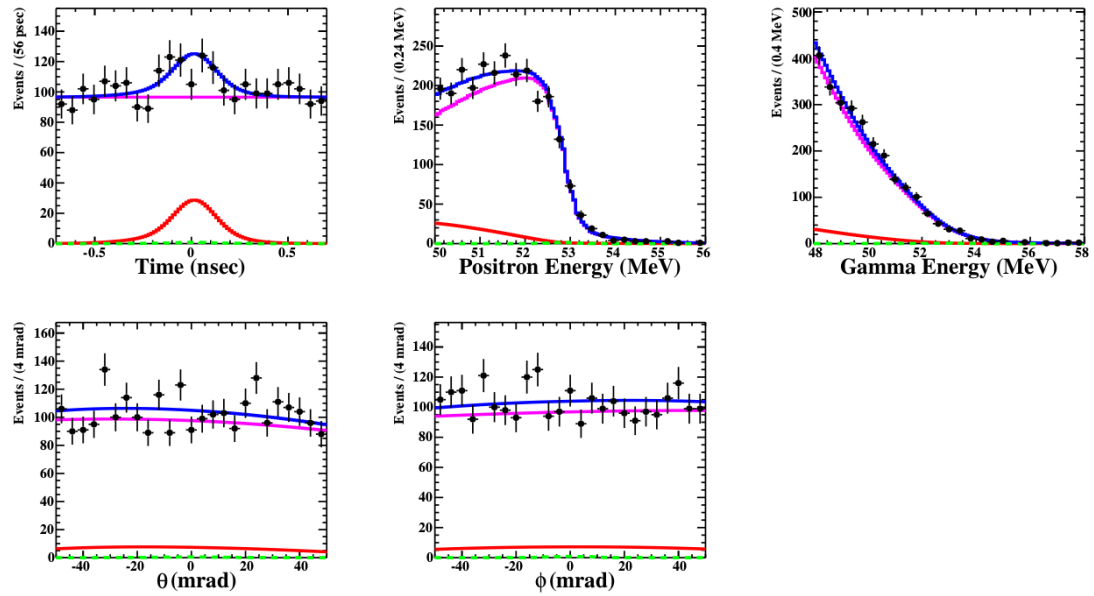

Figure 3. Likelihood fit result for 2009-2011 combined dataset. Data (black points) are shown with the projections of the 5D fit (blue line) on each of the kinematic variables. Individual best fits for signal (green), RMD (red) and accidental background (magenta) are also shown.

\section{The MEG upgrade}

The major modifications of the MEG apparatus [9] are shown in Figure 4: the drift chamber and the timing counters are replaced while the calorimeter undergoes substitutions and rearrangements of the photosensors.

The new positron spectrometer consists of a drift chamber and two pixelated timing counters. In the new configuration positrons traverse less material along their path, and the capabilities of matching the information from the two detectors are improved. The new tracker is a single-volume cylindrical drift chamber, composed of ten layers of approximately squared drift cells with side ranging from $6 \mathrm{~mm}$ (in the innermost layer) to $9 \mathrm{~mm}$ (in the outermost one) at alternating stereo angles $\theta_{s}$ ranging from $\pm 6^{\circ}$ to $\pm 8^{\circ}$. High transparency is achieved using an ultra-low mass gas mixture with helium and isobutane $85: 15$. This is fundamental for keeping the Coulomb multiple scattering at a minimum for the low-momentum positrons from $\mu^{+}$decays. Gold-plated tungsten wires with $20 \mu \mathrm{m}$ diameter are used as anodes, while cathode and guard wires are 40-50 $\mu \mathrm{m}$ silver-plated aluminium wires. The resulting radiation length per track turn is about $1.6 \times 10^{-3} X_{0}$. Due to the large number of hits, a rough determination of each hit's longitudinal coordinate conveys precious information for pattern recognition strategies. For this sake, the readout of wires on both ends will permit to exploit charge division and time propagation difference. The high transparency of the chamber has the drawback of a poor ionisation statistics, which results in a bias and a worsening of resolution in the estimate of the impact parameter in the drift cells. For a clean measurement of the single-hit resolution several drift chamber prototypes were tested in a cosmic ray facility set up at INFN Sezione di Pisa [10]. The measured single-hit resolution is $110 \mu \mathrm{m}$, averaged over a large range of angles and of impact parameters. The longitudinal coordinate of hits will be determined by exploiting the stereo angle, with a resolution $\sigma_{z}=\sigma_{r} / \sin \theta_{s} \approx 1 \mathrm{~mm}$, about a factor 100 better than the $\sim 10 \mathrm{~cm}$ resolution of the $z$ reconstructed with charge division and relative time.

The new pixelated timing counters consist of $2 \times 256$ scintillator tiles $\left(120 \times 50 \times 5 \mathrm{~mm}^{3}\right)$. The pixels are read out at two sides by $6+6$ Silicon PhotoMultipliers (SiPMs) mounted in series connection on a 

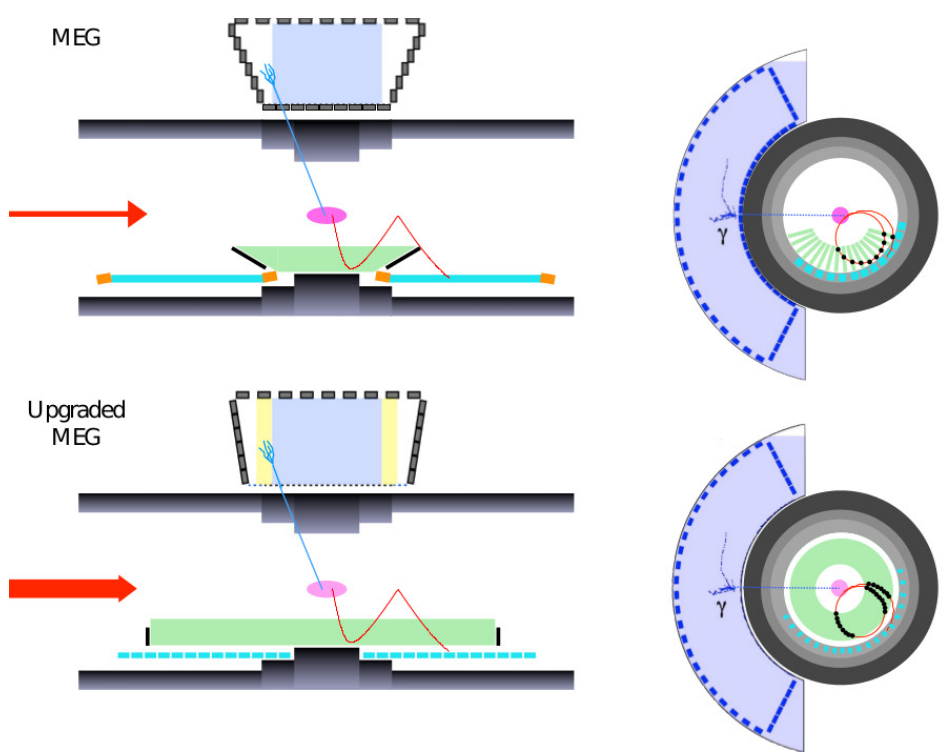

Figure 4. Improvements of the MEG detector. The upgraded apparatus consists of a new tracker with higher resolution and granularity and an improved matching with a new pixelated timing counter. The xenon calorimeter gains a larger acceptance and a better scintillation light collection. See full text for more details.

Printed Circuit Board acting as a frame: this solution keeps the material budget along the positron path to a minimum and maximizes the detector coverage. The segmentation of the timing counters brings an intrinsic potential in improving the timing resolution, coming from the possibility of averaging the positron hit time over the multiple hit pixels $(\sigma \sim 1 / \sqrt{N})$. The average number of tiles hit by a

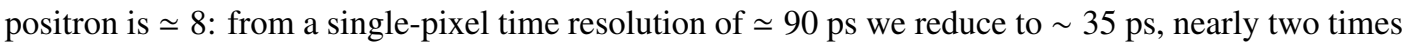
better than previous timing counter.

The main issue of MEG xenon calorimeter is the dependence of resolutions on the depth of the $\gamma$-conversion. This is due to the granularity of the 2" PMTs (with an area of $81 \mathrm{~cm}^{2}$ ) on the front face distributed in a mesh of $6.2 \mathrm{~cm}$ side. In MEG II the PMTs in the entrance face will be replaced by smaller photosensors, $12 \times 12 \mathrm{~mm}^{2}$ SiPMs. Also the imaging power is thus greatly increased and pile up events can be better identified. The layout of the lateral faces will be modified as well in order to avoid shadow areas, which result in a reduced acceptance. The new structure is visible in Figure 4, where the wider acceptance region is highlighted.

In Table 1 the resolutions expected for MEG II are reported. Better detector resolutions will permit a higher beam rate, about $7 \times 10^{7} \mu / \mathrm{s}$. In three years of data taking MEG II expected sensitivity on the branching ratio of $\mu \rightarrow e \gamma$ is about $5 \times 10^{-14}$.

\section{Conclusion}

The final MEG result is soon to be released, with a sensitivity of about $4 \times 10^{-13}$ on the branching ratio. At the same time MEG II is performing an engineering run with a drift chamber mock up and a sector of the timing counter. The mock up drift chamber has the same structure of the final one, but only one layer of anode wires. 
The full apparatus is thus expected to be on beam in Autumn 2016. In three years of data taking the expected sensitivity is $5 \times 10^{-14}$, about ten times better than the current limit set by MEG.

\section{References}

[1] S. M. Bilenky, S. Petcov, and B. Pontecorvo, Phys.Lett., vol. B67, p. 309, 1977.

[2] W. J. Marciano, T. Mori, and J. M. Roney, Ann. Rev. Nucl. Part. Sci., vol. 58, pp. 315-341, 2008.

[3] A. de Gouvêa, P. Vogel, Progress in Particle and Nuclear Physics, vol. 71, pp. 75-92, 2013.

[4] J. Adam and et al., Phys. Rev. Lett., vol. 110, p. 201801, May 2013.

[5] U. Bellgardt et al., Nucl. Phys., vol. B299, p. 1, 1988.

[6] W. H. Bertl et al., Eur. Phys. J., vol. C47, pp. 337-346, 2006.

[7] J. Adam et al., Eur.Phys.J., vol. C73, p. 2365, 2013.

[8] S. Ritt, R. Dinapoli, and U. Hartmann, Nucl. Instrum. Meth., vol. A623, pp. 486-488, 2010.

[9] A. M. Baldini and et al., ArXiv e-prints, 1301.7225, Jan. 2013.

[10] L. Galli, E. Baracchini, S. Bettarini, F. Bosi, E. Cavallaro, S. Dussoni, M. Minuti, F. Morsani, D. Nicolo, G. Signorelli, F. Tenchini, M. Venturini, and J. Walsh, Nuclear Science, IEEE Transactions on, vol. 62, no. 1, pp. 395-402, 2015. 